\title{
Unit commitment based reliability analysis with contingency constraint
}

\author{
Raheema Syed $^{1}$, P. Srinivasa Varma ${ }^{2}$, R. B. R. Prakash ${ }^{3}$, Ch. Rami Reddy ${ }^{4}$ \\ 1,2,3 Department of EEE, Koneru Lakshmaiah Education Foundation, India \\ ${ }^{4}$ Department of EEE, Nalanda Institute of Engineering and Technology, India
}

\begin{tabular}{l}
\hline Article Info \\
\hline Article history: \\
Received Jan 17, 2019 \\
Revised Apr 20, 2019 \\
Accepted May 11, 2019 \\
\hline
\end{tabular}

\section{Keywords:}

Benders decomposition Branch and cut algorithm Contingency constraints Reliability Unit commitment

\begin{abstract}
Unit commitment state's the strategic choice to be prepared in order to define which of the accessible power plants should be taken into account to supply power. It permits utilities to reduce generation price of power. In this paper, the unit commitment problem is elucidated by taking N-1-1 contingency as a foremost constraint. The standard N-1-1 contingency takes the loss of sequential two components in the network having intervening interval for network modifications in the middle of two losses. The crucial objective to carry out contingency constrictions is to make certain that the operations of power system are adequately strong to unexpected losses of the components of the network. The optimal scheduling/allocation of the generating units is resolved by taking into account the $\mathrm{N}-1-1$ criterion of contingency. By considering the N-1-1 criterion of contingency, the problem results to give an optimised model which is a linear model of mixed integer form. The linear program of mixed integer is a technique of an operational assessment in which restriction is imposed on few variables to be integers. Primarily benders decomposition was considered but for the improvement of results, the algorithm of branch and cut is presented. IEEE 30 bus system is taken into consideration and widespread analysis is accomplished to associate performance of the system under N-1-1 criterion contingency. The computational outcomes determine the value for taking into concern the intervening interval for the adjustments of the system with respect to the cost and robustness of the system. Later to the above model reliability assessment is proposed to calculate the Loss Of Load Expected (LOLE). This model is solved using MATLAB/MATPOWER software.
\end{abstract}

Copyright () 2019 Institute of Advanced Engineering and Science. All rights reserved.

\section{Corresponding Author:}

Raheema Syed,

Department of Electrical and Electronics Engineering,

Koneru Lakshmaiah Education Foundation,

Vaddeswaram, Guntur, Andhra Pradesh, India - 522502.

Email: raheemasyed@gmail.com

\section{INTRODUCTION}

The unit commitment implicates the units to be selected which can provide the system with the anticipated load over an essential time interval at least cost. Moreover, offers the spinning reserve which is an operative reserve of specified margin taking into consideration of the power systems constraints for instance least possible time for on/off, up/down ramping limits and capacity of least/extreme generation. Since the probability of multiple components in the system of near failures happening simultaneously is comparably less, taking into account the interval for intervening for the adjustment of the operator may result in least conservative and more decisions of unit commitment which are economical. In recent period, with stable unit commitment decisions, optimum flow of power for N-1-1 constrained contingency could be studied by interdiction technique [1]. Analysis of interdiction implicates that the subset of the components of the system 
are identified such that the loss of load of the system would maximize if interdicted during the worst case. Outages due to potentially cascading in the course of the contingencies can be avoided by modelling the adjustments of the system optimally. Basically contingency constraints make sure that even during the failure of a single component the performance of the system will continue 'as is'. In the case of failure of multiple components during the contingency constraints, it the required that the system performance continues at an level which is acceptable with shedding of load of some amount and during the recovery interval overloading of system is permitted. Due to presence of contingency in power system there might be an unbalance amongst supply and demand.

Contingency due to N-1-1 specifies that either the unit of generation or a line of transmission has a loss which is followed by the adjustments of the system. At an advanced interval of time there is an additional loss of a line or a generator in the system. Therefore contingency due to N-1-1 has three various interval of time namely, base case followed by primary contingency and later secondary contingency. During the normal condition, the system functions with no component failure which is referred to as the base case. When there is a failure of a single component then the system functions as the primary contingency. At $t_{1}$, there is a change in state from nominal operation to operation due to contingency and primary loss is also observed. During the secondary contingency, operation of the system with the failure of two components is observed and it's acceptable for precise shedding of load and overloading of line [2]. In references [3, 4] consideration of unit commitment with $\mathrm{N}-1$ constrained contingency is taken into account and employed switching of line to ease congestion so as to produce resources of generation which can be dispatched economically. In recent times [5], advancement of a robust model of unit commitment has been taken place depending on N-k constrained contingency and ambiguity of interval in view of uninterrupted wind/load. The solution of the model is obtained by combination of modified method of benders decomposition and algorithm of generation by column and constraint (C\&CG). As the demand and renewable penetration has increased, the importance for analysing the contingencies has also increased. In few circumstances there is less redundancy due to the increase in demand. In [6], a new criterion N-k-E was presented where the demand of the system must be met by no less than $\left(1-\varepsilon^{j}\right)$ fraction following the component of system failure of $j$. Two different algorithms were proposed namely benders decomposition and contingency screening implicitly. Reference [7], ambiguous sources of renewable energy with a framework of probabilistic failure unit commitment constrained is described where for stable set of outages for transmission and generator, the probabilities of failure are exhibited as unambiguous functions for the variables of binary scheduling and on loss of load expected the penalties are imposed. For day ahead market, unit commitment consisting of reliability constrains is presently [8] for combined energy and scheduling of reserve where if there is some instantaneous loss in the unit generation then demand needs to be fulfilled with certain probability. A stochastic model of chance constrained two stage for solving the problem of unit commitment with two $n-k$ criteria for security is presented. Reference [9] presents optimization framework for two stage under contingency N-k constraints for combined energy and scheduling of reserve. Benders decomposition approach with inequalities for tighter formulation is presented. Previously, Benders decomposition was one of the solution approach used to solve the linear programming for mixed integers model for unit commitment. The foremost concept of bendersdecomposition is the formation of a main problem and a sub divided problem from the problem which sequentially gets divided into sub problems which are independent. It solves the linear program of mixed integer in an algorithm which is an iterative process because extensive form thru direct solution is not practical. The benders decomposition has a drawback that is execution of the algorithm is easy but its computational controllability is restricted since the main problem needs to be solved at each iteration so as to select the unit commitment candidate decision. Therefore to overcome this drawback an algorithm known as branch and cut is outlined [10]. In [11], one of the approach to solve linear program for mixed integers is branch and cut and its principle is, a solution containing smaller subset is obtained from a total feasible solution set. Evaluation for these smaller subset can be performed systematically. For larger problems of combinatorial the computational time reduces. Unit commitment problem is solved in an hourly manner incorporating various constraints of transmission lines and units of generation, uncertainty in forecasting of load, random outages of components into reliability problem. In the stochastic unit commitment, one of the constraints expected loss of load is included so as to calculate the cost required for the reserve to be supplied [12]. The availability of capacity of power at a load bus is assessed using reliability calculation in a composite system [13].

In this paper, N-1-1 contingency for solving the unit commitment problem is considered. The vital difference between this model and previous models is the consideration of the time for intervening in the middle of the primary and secondary contingencies. The unit commitment problem with contingency N-1-1 constraints is solved using an algorithm of branch and cut. Lastly, reliability evaluation is proposed to the above model. The LOLE is calculated. Reliability evaluation is carried out for two reasons. Primarily, longterm assessments are carried out to support in the planning of the system. Next, short-term valuations help in 
day to day decisions for operation. The model proposed here for reliability analysis based N-1-1 contingency constrained unit commitment has not been analysed previously and this model has advantage over the other models because of the intervening time for system adjustment and additionally the adequacy evaluation for generating capacity is also evaluated.This manuscript is organised as follows: Initially in this section the introduction and literature survey is discussed. Next in Section 2, the mathematical formulation for contingency N-1-1 constrained unit commitment is discussed. Section 3 presents the reliability evaluation. In Section 4 the results are analysed and in Section 5 the conclusion for work done is discussed [14].

\section{MATHEMATICAL FORMULATION}

As a part of mathematical formulation, under the circumstances of the contingency scenario, the decisions of unit commitment is made. The mathematical formulation is given below [10, 15]:

\subsection{Formulation for Baseline Unit Commitment}

$$
\min _{x, f, p, \theta} c^{s}(x)+c^{p}(p)
$$

Such that $\mathrm{x} \in \mathcal{X}$

$\mathrm{Hp}^{\mathrm{t}}+\mathrm{Af}^{\mathrm{t}}=\mathrm{d}^{\mathrm{t}}, \forall \mathrm{t}$

$B_{e}\left(\theta_{i}^{t}-\theta_{j}^{t}\right)-f_{e}^{t}=0, \forall e=(i, j), t$

$\left|\mathrm{f}^{\mathrm{t}}\right| \leq \overline{\mathrm{f}}, \forall \mathrm{t}$

$$
\underline{\mathrm{p}_{\mathrm{g}}} \mathrm{x}_{\mathrm{g}}^{\mathrm{t}} \leq \mathrm{p}_{\mathrm{g}}^{\mathrm{t}} \leq \overline{\mathrm{p}_{\mathrm{g}}} \mathrm{x}_{\mathrm{g}}^{\mathrm{t}}, \forall \mathrm{g}, \mathrm{t}
$$

$$
\underline{\mathrm{r}_{\mathrm{g}}}\left(\mathrm{x}_{\mathrm{g}}\right) \leq \mathrm{p}_{\mathrm{g}}^{\mathrm{t}}-\mathrm{p}_{\mathrm{g}}^{\mathrm{t}-1} \leq \overline{\mathrm{r}}_{\mathrm{g}}\left(\mathrm{x}_{\mathrm{g}}\right), \forall \mathrm{g}, \mathrm{t}
$$

Where, $B_{\mathrm{e}}$ : Line e's susceptance

$\bar{f}$ : Capacities of a line vector

$\mathrm{d}^{\mathrm{t}}$ : Demand vector in interval $\mathrm{t}$

$\mathrm{p}_{\mathrm{g}}, \overline{\mathrm{p}_{\mathrm{g}}}$ : Lower and upper limits for the capacity of a generator $\mathrm{g}$

$\overline{\mathrm{r}_{\mathrm{g}}}(\mathrm{x}), \overline{\mathrm{r}_{\mathrm{g}}}(\mathrm{x})$ : Ramp down/up rates vector for the vector of the committed units $\mathrm{x}$

$\overline{\mathrm{H}}$ and $\mathrm{A}$ : Incidence matrix

$\mathrm{p}_{\mathrm{g}}^{\mathrm{t}}, \mathrm{f}_{\mathrm{e}}^{\mathrm{t}}, \theta_{\mathrm{i}}^{\mathrm{t}}$ : The level for generation for $\mathrm{g}$ unit, flow of power on line $\mathrm{e}$ and the angle on bus $\mathrm{i}$ in interval $\mathrm{t}$ when there is no contingency

In objective function i.e., in (1) the sum of the cost of the start-up and cost for shutdown $\mathrm{c}^{\mathrm{s}}(\mathrm{x})$ and cost for generationc $\mathrm{c}^{\mathrm{p}}(\mathrm{p})$ is being minimized. Here ' $\mathrm{p}$ ' is prescribed as the dispatch levels and for thermal generation units the cost for generation is generally specified as quadratic function. Constraint (2) gives the information regarding the lowest uptime and downtime for a generators necessity and the cost for start-up and shutdown is calculated as the function for the committed units. This term $\mathcal{X}$ symbolizes the set of possibility. Constraint (3) gives the steadiness of power at bus individually. Constraint (4) gives information regarding the flow of power on a line. Constraint (5) gives the limits for the capacity for the line of transmission. Constraint (6) denotes the limits for lower and upper dispatch of generator. Constraint (7) denotes the limits for ramping of a generator between the two sequential intervals of time. The reformulation for the linear program of mixed integer can be obtained by linearizing in piecewise manner the quadratic function of cost for generation for the problem of baseline unit commitment.

\subsection{Contingency Modelling}

$$
\begin{aligned}
& \mathcal{C}=\left\{\mathrm{c} \in\{0,1\}^{(\mathrm{G}+\mathrm{E}) \times \mathrm{T}}\right. \\
& \sum_{\mathrm{g} \in \mathcal{G}} \mathrm{c}_{\mathrm{g}}^{\mathrm{t}} \sum_{\mathrm{e} \epsilon \mathcal{E}} \mathrm{c}_{\mathrm{e}}^{\mathrm{t}} \leq 1, \forall \mathrm{t} \in \mathrm{T}
\end{aligned}
$$




$$
\begin{aligned}
& \sum_{\mathrm{t} \epsilon \mathrm{T}} c_{\mathrm{g}}^{\mathrm{t}} \leq 1, \forall \mathrm{g} \in \mathcal{G} \\
& \sum_{\mathrm{t} \epsilon \mathrm{T}}^{\mathrm{t}} \leq 1, \forall \mathrm{e} \in \varepsilon \\
& \left.\sum_{\mathrm{t} \in \mathrm{T}} \sum_{\mathrm{g} \in \mathcal{G}} \mathrm{c}_{\mathrm{g}}^{\mathrm{t}}+\sum_{\mathrm{t} \in \mathrm{T}} \sum_{\mathrm{e} \in \varepsilon} \mathrm{c}_{\mathrm{e}}^{\mathrm{t}}=2\right\}
\end{aligned}
$$

Where, $\mathcal{G}$ : Generator set, indexed by $\mathrm{g}$

E: The transmission line set

$\mathrm{T}$ : The time period set, indexed by $\mathrm{t}$

Here, $\mathcal{C}$ is a set for all contingency N-1-1 situations taking into account all the possible interval of periods for the losses in primary and secondary and units of generating and lines of transmission. As $\mathrm{c} \in \mathcal{C}$, following identifications are made, firstly $t_{1}$ and $t_{2}$ are the two intervals of time denoting the period for the losses of primary and secondary respectively. Secondly, $c_{g}^{t_{1}}=1$ or $c_{e}^{t_{1}}=1$ and $c_{g}^{t_{2}}=1$ or $_{e}^{t_{2}}=1$ represents a component which is failed respectively in individual time period. In the set of the constraints (8)(12), states the failure of two individual components in different period of time (12) where $t 1$ and $t 2$ are the initial failure period and failure for second period. Constraint (9) denotes that maximum failure of one component can take place in any period of time. Constraints (10) and (11) denotes that the failure of individual component happens maximum one time. Constraint (12) requires the failure of two components precisely.

Precise shedding of load and overloading of line are allowable during the period for secondary contingency. To ease the infeasibility of operation the factor for overloading of line $\mathrm{o}_{\mathrm{e}}$ and allowable shedding of load $\varepsilon$ can be employed. Three different quantities are presented that is ' $w$ ' which indicates in contingency, ' $h$ ' denotes the loss of load which is allowable and 'o' denotes the overloading of line.

$$
\begin{aligned}
& \mathrm{w}_{\mathrm{e}}^{\mathrm{ct}}=\sum_{\tau=1}^{\mathrm{t}} \mathrm{c}_{\mathrm{e}}^{\mathrm{ct}}, \forall \mathrm{e}, \mathrm{t} \\
& \mathrm{w}_{\mathrm{g}}^{\mathrm{ct}}=\sum_{\tau=1}^{\mathrm{t}} \mathrm{c}_{\mathrm{g}}^{\mathrm{ct}}, \forall \mathrm{g}, \mathrm{t} \\
& \mathrm{h}_{\mathrm{i}}^{\mathrm{ct}}=\left\{\begin{array}{cc}
0 & \forall \mathrm{i}, \mathrm{t}=1, \ldots, \mathrm{t}_{2}^{\mathrm{c}}-1 \\
\mathrm{~d}_{\mathrm{i}}^{\mathrm{t}} & \forall \mathrm{i}, \mathrm{t}=\mathrm{t}_{2}^{\mathrm{c}}, \ldots, \mathrm{T}
\end{array}\right. \\
& \mathrm{o}_{\mathrm{e}}^{\mathrm{ct}}=\left\{\begin{array}{cc}
0 & \forall \mathrm{e}, \mathrm{t}=1, \ldots, \mathrm{t}_{2}^{\mathrm{c}}-1 \\
\mathrm{o}_{\mathrm{e}} & \forall \mathrm{e}, \mathrm{t}=\mathrm{t}_{2}^{\mathrm{c}}, \ldots, \mathrm{T}
\end{array}\right.
\end{aligned}
$$

Constraints (15) and (16) needs the loss of load allowable and the factor for overloading are equated to $d_{i}^{t}$ and $o_{e}$ at the time of secondary contingency. During the remaining interval it has zero value.

\subsection{Contingency N-1-1 Constrained Unit Commitment Model}

$$
\begin{aligned}
& \min \\
& x, f, p, \theta \quad c^{c}(x)+c^{p}(p) \\
& \mathrm{f}^{\mathrm{c}}, \mathrm{p}^{\mathrm{c}}, \mathrm{q}^{\mathrm{c}}, \theta^{\mathrm{c}} \\
& \text { Such that Constraints (2)-(7) } \\
& \mathrm{Hp}^{\mathrm{ct}}+\mathrm{Af}^{\mathrm{ct}}+\mathrm{q}^{\mathrm{ct}}=\mathrm{d}^{\mathrm{t}}, \forall \mathrm{t} \\
& B_{e}\left(\theta_{i}^{c t}-\theta_{j}^{c t}\right)\left(1-w_{e}^{c t}\right)-f_{e}^{c t}=0, \forall e=(i, j), t \\
& \left|\mathrm{f}_{\mathrm{e}}^{\mathrm{ct}}\right| \leq \overline{\mathrm{f}}_{\mathrm{e}}\left(1-\mathrm{w}_{\mathrm{e}}^{\mathrm{ct}}\right)\left(1+\mathrm{o}_{\mathrm{e}}^{\mathrm{ct}}\right), \forall \mathrm{e}, \mathrm{t} \\
& \underline{p_{g}} x_{g}^{t}\left(1-w_{g}^{c t}\right) \leq p_{g}^{c t} \leq \overline{p_{g}} x_{g}^{t}\left(1-w_{g}^{c t}\right), \forall g, t \\
& \underline{r_{g}}\left(x_{g}\right)\left(1-w_{g}^{c t}\right) \leq p_{g}^{c t}-p_{g}^{c t-1} \leq \overline{r_{g}}\left(x_{g}\right)\left(1-w_{g}^{c t}\right), \forall g, t \\
& \mathrm{q}^{\mathrm{ct}} \leq \mathrm{h}^{\mathrm{ct}}, \forall \mathrm{t}
\end{aligned}
$$


Where $\mathrm{p}_{\mathrm{g}}^{\mathrm{ct}}, \mathrm{f}_{\mathrm{e}}^{\mathrm{ct}}, \theta_{\mathrm{i}}^{\mathrm{ct}}$ are the level of generation for $\mathrm{g}$ unit, flow of power on line $\mathrm{e}$, angle on bus ' $\mathrm{i}$ ' in interval $\mathrm{t}$ when contingency is present.

The above model is a linear program of mixed integer in tremendously large scale. The objective (17) comprises of the generation cost when there is no contingency and the cost of the unit commitment. (19) gives the steadiness of power at bus individually with demand $q^{c t}$ which is unsatisfied. (20) gives information regarding the flow of power on a line. Constraint (21) gives the limits for the capacity for the line of transmission with the factor of overloading when contingency takes place at the secondary interval. Constraint (22) denotes the limits for lower and upper dispatch of generator. Constraint (23) denotes the limits for ramping of a generator. Higher limit on loss of load at bus individually (24).

\subsection{Branch and Cut Algorithm}

Due to the improvement in technologies, the optimization solver made simpler the operation for the algorithm of branch and cut. Since the inequalities are added directly within the tree of branch and bound the necessity to explore repeatedly the branch and bound tree can be avoided, defined by the variable $\mathrm{x}$ of binary unit commitment. It includes all the constraints which are dependent of time and doesn't need any ordering of units prior.The relaxation for linear program of main problem be:

$$
\min _{x, f, p, \theta} c^{s}(x)+c^{p}(p)
$$

Such that $\mathrm{x} \in \mathcal{X}$

$0 \leq \mathrm{x} \leq 1$

(3) - (7)

This technique is used when there is no constraint containing integer value for the solution of linear program by means of a simplex algorithm. Suppose a result which is optimum is obtained and instead of integer variable there is a value which is non-integer, an algorithm known as cutting plane could be considered so as to find the constraints which are linear. At the time of branch and bound, the upper limit of linear program relaxation is taken by the solution which is non-integral and the lower limit is served by integral solution. In case an upper limit is lesser than present limit which is less, then a node could be pruned. The brief algorithm:

1. Initial integer linear program is added to the active problems list, $\mathrm{L}$

2. $\mathrm{x}^{*}$ is set as null and $\mathrm{v}^{*}$ is set as negative infinity

3. While the list of active problem, L isn't empty

a. A problem needs to be selected and removed from $\mathrm{L}$

b. Linear program relaxation is solved.

c. In case it's an infeasible solution, head back to 3 (while), or else represent $\mathrm{x}$ for solution and $\mathrm{v}$ as objective value.

d. In case $\mathrm{v} \leq \mathrm{v}^{*}$, head to 3 .

e. In case if the integer is $\mathrm{x}$, set $\mathrm{x}^{*} \leftarrow \mathrm{x}$ and $\mathrm{v}^{*} \leftarrow \mathrm{v}$ and head to 3 .

f. If desired, then the cutting planes which are disrupted by $x$ are searched. If such any violation, need to include them in linear program relaxation and get back to $3 \mathrm{~b}$.

g. The problem is partitioned by branching with feasible regions restriction. Include this problem to $\mathrm{L}$, head to 3 .

4. Return $x^{*}$

\section{RELIABILITY EVALUATION}

Reliability denotes the supply of energy uninterruptedly to end consumers when considering outages of scheduled and unscheduled. Due to contingency, the supply and demand might face some unbalance between them. Loss of load expected is the anticipation that the supply generation available will not meet the load of the system hourly. Also defined as number of days expected that the system would be unsuccessful to supply demand of load. LOLE is generally represented in units of time period in place of values which are in percentage. If the result for expected loss of load is greater, then it can be concluded that it has deficiencies in charging of power or the units which are present are disposed poorly. Calculation for LOLE:The utmost appropriate technique is signifying the power system in tabular form which comprises of availabilities, un-availabilities and capacities. Generally, a diagram of loads of daily basis is given for consideration. 
Cumulative probabilities are calculated from the probabilities of combinations of unit outage. Index for LOLE is given as a product of the duration of the load expected and the probability of cumulative outage for the states essential. LOLE is expressed in hours/year when considering the curve for everyday hourly load variation on yearly basis.

$$
\text { LOLE }=\sum_{\mathrm{i}=1}^{\mathrm{n}} \mathrm{p}_{\mathrm{i}} \mathrm{t}_{\mathrm{i}}
$$

Where $p_{i}$ is the probability of outage for individual capacity and $t_{i}$ is the loss of supply for the duration in days.

\section{RESULTS AND ANALYSIS}

An investigation of decisions for dispatch and optimum commitment for IEEE 30 bus system across the various criteria of contingency is analysed. In Table 1 the load profile for 12 hours is mentioned.

\begin{tabular}{cc} 
Table 1. Load Profile for 12 Hours \\
Hour & Demand (MW) \\
\hline 1 & 166 \\
2 & 196 \\
3 & 229 \\
4 & 267 \\
5 & 283.4 \\
6 & 272 \\
7 & 246 \\
8 & 213 \\
9 & 192 \\
10 & 161 \\
11 & 147 \\
12 & 160 \\
\hline
\end{tabular}

Figure 1, 2 and 3 denotes the optimal dispatch levels across various contingencies. The Figure 1 presents the optimum level of dispatch for N-1 contingency. It can be analysed that the generator 1 is in the OFF state during the $1^{\text {st }}, 2^{\text {nd }}, 3^{\text {rd }}, 8^{\text {th }}, 9^{\text {th }}, 10^{\text {th }}, 11^{\text {th }}$ and $12^{\text {th }}$ hour. Generator 2 is in the OFF state during the $1^{\text {st }}$, $2^{\text {nd }}, 8^{\text {th }}, 9^{\text {th }}, 10^{\text {th }}, 11^{\text {th }}$ and $12^{\text {th }}$ hour. While the remaining other generators are all in the ON state for all the hours and the dispatch levels varies accordingly for each generator.

The Figure 2 shows the optimum level of dispatch for the N-1-1 contingency. It can be analysed that the generator 1 is in the OFF state during the $1^{\text {st }}, 2^{\text {nd }}, 3^{\text {rd }}, 7^{\text {th }}, 8^{\text {th }}, 9^{\text {th }}, 10^{\text {th }}, 11^{\text {th }}$ and $12^{\text {th }}$ hour. Generator 2 is in the OFF state during the $1^{\text {st }}, 2^{\text {nd }}, 8^{\text {th }}, 9^{\text {th }}, 10^{\text {th }}, 11^{\text {th }}$ and $12^{\text {th }}$ hour. While the remaining other generators are all in the ON state for all the hours and the dispatch levels varies accordingly for each generator.

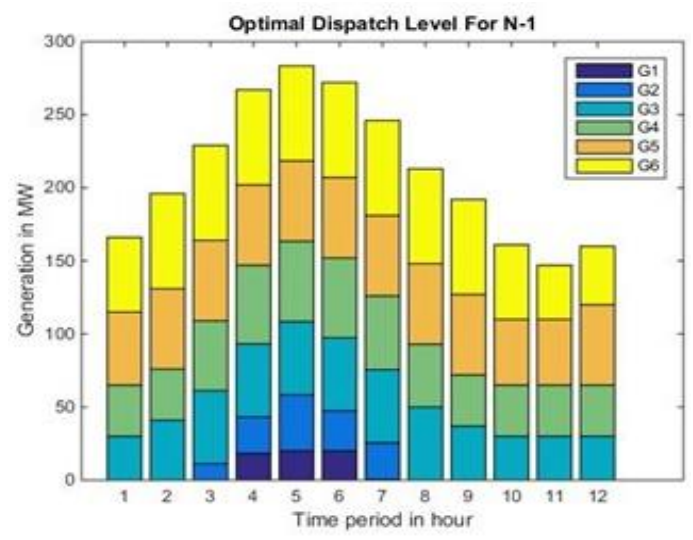

Figure 1. Optimal dispatch level for N-1 contingency

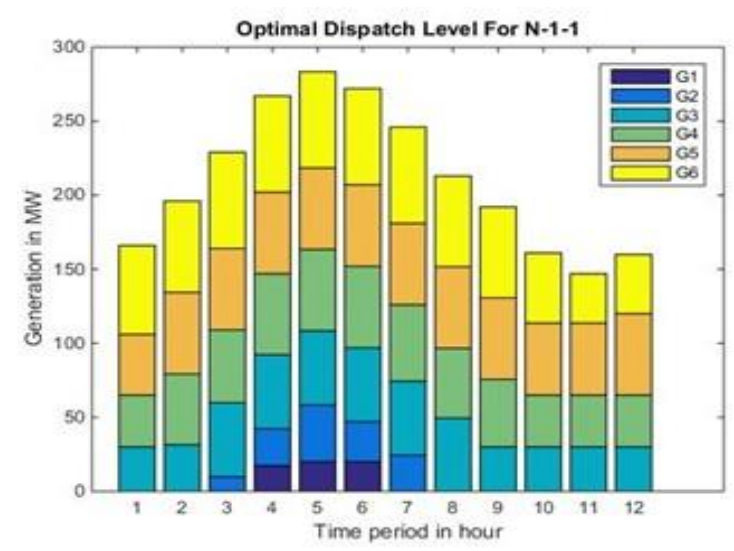

Figure 2. Optimal dispatch level for N-1-1 contingency 
Figure 3 presents the optimal dispatch level for N-2 contingency. It can be analysed that the generator 1 is in the OFF state during the $1^{\text {st }}, 2^{\text {nd }}, 8^{\text {th }}, 10^{\text {th }}, 11^{\text {th }}$ and $12^{\text {th }}$ hour. Generator 2 is in the OFF state during the $1^{\text {st }}, 10^{\text {th }}, 11^{\text {th }}$ and $12^{\text {th }}$ hour. While the remaining other generators are all in the ON state for all the hours and the dispatch levels varies accordingly for each generator.

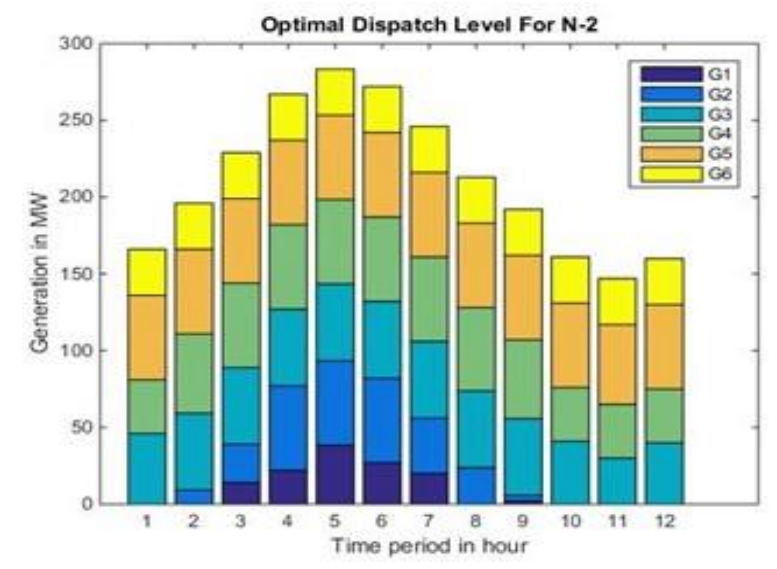

Figure 3. Optimal dispatch level for N-2 contingency

Furthermore, the Figures 1, 2 and 3 denote's the units committed and their dispatch levels in the 12 hours' time period. All the units of generator 3, 4, 5, 6 are committed at all hours for the above various contingencies. At the $5^{\text {th }}$ hour there is maximum generation and when its $11^{\text {th }}$ hour the generation is the least. The dispatch levels varies for different generators at different hour period for different contingencies.

Next, in Figure 4 comparison is made for total system costs across various criteria of contingency for IEEE 30 bus system. The cost for $\mathrm{N}-1, \mathrm{~N}-1-1$ and $\mathrm{N}-2$ contingencies are $\$ 53142, \$ 53361$ and $\$ 69351$. It can be concluded that the cost rises uniformly across the contingency constraints $\mathrm{N}-1, \mathrm{~N}-1-1$ to $\mathrm{N}-2$. The difference in the cost across the N-2 and N-1-1 contingency criteria exhibit the value for the time of intervening for the adjustment of the system for the duration of a multiple-failure scenario across contingency

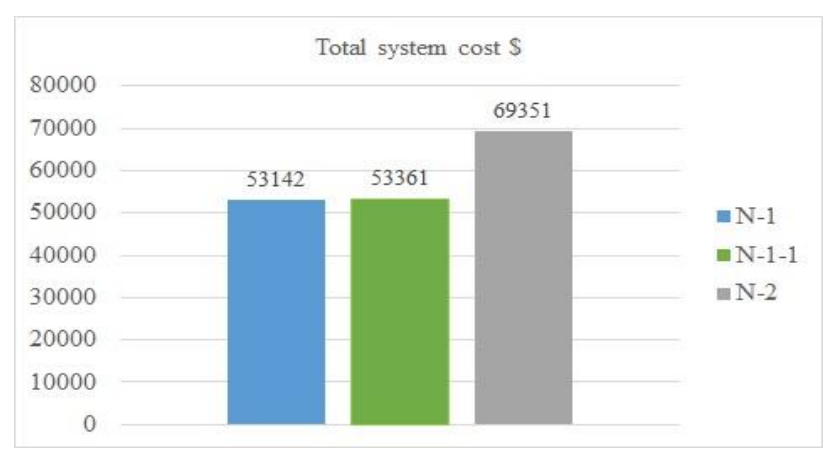

Figure 4. Total system cost under various contingencies

Lastly, in Figure 5 comparison is made for the expected loss of load across different contingency analysis. The loss of load for N-1-1 is $3.788 \mathrm{hr} /$ year and for N-2 it is $3.829 \mathrm{hr} /$ year. As a result, the loss of load for $\mathrm{N}-2$ is more compared to $\mathrm{N}-1-1$ because for $\mathrm{N}-2$ simultaneous failure takes place while for $\mathrm{N}-1-1$ there is an intervening time for system adjustment. If LOLE is more, then the system cost will also increase and vice versa. Henceforth from results it can be concluded that for N-2 contingency the loss of load and system cost is high as compared to $\mathrm{N}-1-1$ contingency. In $\mathrm{N}-1-1$, having a time interval for system adjustment is better to avoid the losses. 


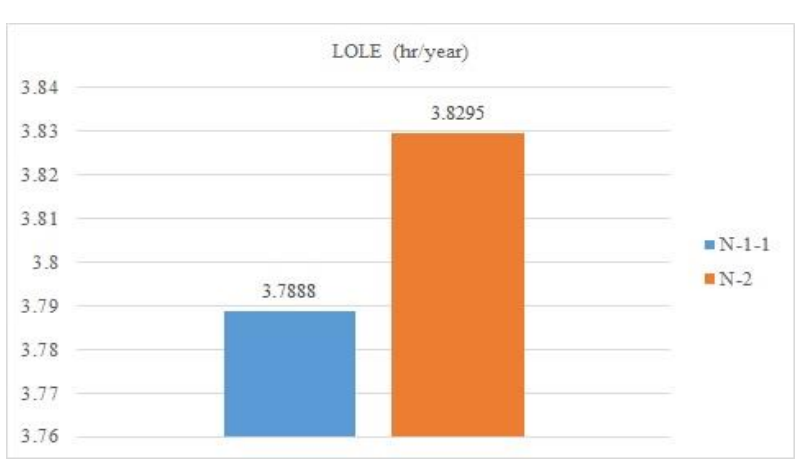

Figure 5. Loss of load expected for IEEE 30 bus

\section{CONCLUSION}

A model of unit commitment taking into account the non-simultaneous failures of components with the time for intervening by operators for the adjustment of the system is taken into consideration. This model gives a large scale linear program of mixed integer. To solve such model branch and cut algorithm is used. The dispatch levels of the system with associated cost of generation across various contingency constraints are evaluated and presented. Furthermore to the above evaluation, reliability assessment is presented to calculate the expected loss of load for the unit commitment problem on the hourly basis. Comparison is made for the results obtained across different contingency and hence it's concluded that having an intervening time for system adjustment is better with respect to cost and expected loss of load.

\section{REFERENCES}

[1] N. Fan, R. Chen, and J.-P. Watson, "N-1-1 contingency-constrained optimal power flow by interdiction methods," in Proc. 2012 IEEE Power \& Energy Soc. General Meeting, San Diego, CA, USA, Jul. 2012, pp. 1-6.

[2] R. Scott and P. E. Dahman, "N-1-1 contingency analysis using Power World simulator," Power World Corp., Champaign, IL, USA, 2010.

[3] K. W. Hedman, M. C. Ferris, R. P. O’Neill, E. B. Fisher, and S. S. Oren, "Co optimization of generation UC and transmission switching with N-1 reliability,” IEEE Trans. Power Syst.vol. 24, no. 2, pp. 1052-1063, May 2010.

[4] R. P. O'Neill, K.W. Hedman, E. R. Krall, A. Papavasiliou, and S. S. Oren, "Economic analysis of the N-1 reliable UC and transmission switching problem using duality concepts," Energy Syst.vol. 1, no. 2, pp. 165-195, 2010.

[5] B. Hu and L. Wu, "Robust SCUC considering continuous/discrete uncertainties and quick-start units: A two-stage robust optimization with mixed integer recourse," IEEE Trans. Power Syst.vol. 31, no. 2, pp. 1407-1419, Mar. 2016.

[6] R. L.-Y. Chen, A. Cohn, N. Fan, and A. Pinar, "Contingency-risk informed power system design," IEEE Trans. Power Syst.vol. 29, no. 5, pp. 2087- 2096, Sep. 2014.

[7] R. Fern'andez-Blanco, Y. Dvorkin, and M. Ortega-Vazquez, "Probabilistic security-constrained unit commitment with generation and transmission contingencies," IEEE Trans. Power Syst., to be published.

[8] D. Pozo and J. Contreras, "A chance-constrained unit commitment with an n-K security criterion and significant wind generation," IEEE Trans.Power Syst.vol. 28, no. 3, pp. 2842-2851, Aug. 2013.

[9] A. Street, A. Moreira, and J. M. Arroyo, "Energy and reserve scheduling under a joint generation and transmission security criterion: An adjustable robust optimization approach," IEEE Trans. Power Syst.vol. 29, no. 1, pp. 3-14, Jan. 2014.

[10] Zhaomiao Guo, Richard Li-Yang Chen, Neng Fan, Jean-Paul Watson, "Contingency-Constrained Unit Commitment With Intervening Time for System Adjustments," IEEE Trans. Power Syst. vol. 32 , no. 4 , pp. 3049 3059, July 2017.

[11] N. P. Padhy, "UC-A bibliographical survey," IEEE Trans. Power Syst.vol. 19, no. 3, pp. 1196-1205, Aug. 2004.

[12] Lei Wu, Mohammad Shahidehpour, Tao Li, "Cost of Reliability Analysis Based on Stochastic Unit Commitment,"IEEE Trans. Power Syst. vol. 23, no. 3, pp. 1364-1374, Aug. 2008.

[13] T. Bharath Kumar, O. Chandra Sekhar, M. Ramamoorty, and S. V. N. L. Lalitha, "Evaluation of Power Capacity Availability at Load Bus in a Composite Power System," IEEE Journal of Emerging and Selected Topics in Power Electronics. Vol. 4, no. 4 , pp. 1324 - 1331, Oct. 2016.

[14] Sreenivasulu, J., Varma, P. S., \& Sankar, V. (2018). "Selection of renewable power generation to reduce transmission congestion." Paper presented at the 2017 International Conference on Energy, Communication, Data Analytics and Soft Computing, ICECDS 2017, 2048-2053.

[15] M. Carri' on and J. M. Arroyo, "A compuationally efficient mixed-integer linear formulation for the thermal UC problem,” IEEE Trans. Power Syst. vol. 21, no. 3, pp. 1371-1378, Aug. 2006. 